\title{
Complement Plays a Critical Role in Inflammation-Induced Immunoprophylaxis Failure in Mice
}

\section{OPEN ACCESS}

Edited by:

Guido Moll,

Charité-Universitätsmedizin Berlin,

Germany

Reviewed by:

Ellen Van Der Schoot,

Sanquin Research, Netherlands

Lubka T. Roumenina,

INSERM U1138 Centre de Recherche des Cordeliers (CRC), France

*Correspondence:

Jeanne E. Hendrickson jeanne.hendrickson@yale.edu

Specialty section:

This article was submitted to Alloimmunity and Transplantation,

a section of the journal

Frontiers in Immunology

Received: 01 May 2021 Accepted: 14 June 2021

Published: 25 June 2021

Citation:

Escamilla-Rivera $V$ Santhanakrishnan M, Liu J, Gibb DR, Forsmo JE, Foxman EF, Eisenbarth SC, Luckey CJ, Zimring JC, Hudson KE, Stowell SR and Hendrickson JE (2021) Complement Plays a Critical

Role in Inflammation-Induced Immunoprophylaxis Failure in Mice.

Front. Immunol. 12:704072. doi: 10.3389/fimmu.2021.704072
Vicente Escamilla-Rivera ${ }^{1}$, Manjula Santhanakrishnan ${ }^{1}$, Jingchun Liu ${ }^{1}$, David R. Gibb ${ }^{2}$, James E. Forsmo ${ }^{1,3}$, Ellen F. Foxman ${ }^{1,4}$, Stephanie C. Eisenbarth ${ }^{1,4}$, C. John Luckey ${ }^{5}$, James C. Zimring ${ }^{5}$, Krystalyn E. Hudson ${ }^{6}$, Sean R. Stowell ${ }^{7,8}$ and Jeanne E. Hendrickson ${ }^{1,9 *}$

${ }^{1}$ Department of Laboratory Medicine, Yale University School of Medicine, New Haven, CT, United States, ${ }^{2}$ Department of Pathology \& Laboratory Medicine, Cedars-Sinai Medical Center, Los Angeles, CA, United States, ${ }^{3}$ Department of Biomedical Engineering, Georgia Institute of Technology, Atlanta, GA, United States, ${ }^{4}$ Department of Immunobiology, Yale University School of Medicine, New Haven, CT, United States, ${ }^{5}$ Department of Pathology, University of Virginia, Charlottesville, VA, United States, ${ }^{6}$ Department of Pathology and Cell Biology, Columbia University Irving Medical Center, New York, NY, United States,

7 Department of Pathology, Brigham and Women's Hospital, Joint Program in Transfusion Medicine, Harvard Medical School, Boston, MA, United States, ${ }^{8}$ Beth Israel Deaconess Medical Center, Harvard Glycomics Center, Harvard Medical School, Boston, MA, United States, ${ }^{9}$ Department of Pediatrics, Yale University School of Medicine, New Haven, CT, United States

Complement impacts innate and adaptive immunity. Using a model in which the human KEL glycoprotein is expressed on murine red blood cells (RBCs), we have shown that polyclonal immunoprophylaxis (KELIg) prevents alloimmunization to transfused RBCs when a recipient is in their baseline state of heath but with immunoprophylaxis failure occurring in the presence of a viral-like stimulus. As complement can be detected on antibody coated KEL RBCs following transfusion, we hypothesized that recipient complement synergizes with viral-like inflammation to reduce immunoprophylaxis efficacy. Indeed, we found recipient C3 and C1q were critical to immunoprophylaxis failure in the setting of a viral-like stimulus, with no anti-KEL IgG alloantibodies generated in

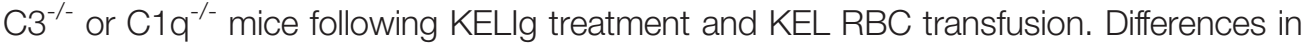
RBC uptake were noted in mice lacking C3, with lower consumption by splenic and peripheral blood inflammatory monocytes. Finally, no alloantibodies were detected in the setting of a viral-like stimulus following KELIg treatment and KEL RBC transfusion in mice lacking complement receptors $\left(\mathrm{CR} 1 / 2^{--}\right)$, narrowing key cells for immunoprophylaxis failure to those expressing these complement receptors. In-vitro studies showed complement fixed opsonized RBCs were significantly less likely to bind to B-cells from $\mathrm{CR} 1 / 2^{-/-}$than wild type mice, potentially implicating lowered B-cell activation threshold in the presence of complement as being responsible for these findings. We thus propose a two-hit model for inflammation-induced immunoprophylaxis failure, where the first "hit" is recipient inflammation and the second "hit" is complement production/sensing. These results may have translational relevance to antigen-antibody interactions in humans.

Keywords: red blood cell, complement, antibody, transfusion, alloimmune 


\section{INTRODUCTION}

Alloimmunization to red blood cells (RBCs) can be significant in transfusion, transplantation, and pregnancy settings. Polyclonal anti-D (RhIg) immunoprophylaxis has been used for over half a century as an effective preventive measure for alloimmunization to $\mathrm{RBC}$ expressing the RhD antigen in pregnancy (1). Immune regulation by antibody-antigen interactions is well known in different immunological settings; however, the mechanism(s) by which RhIg works remains controversial. Further, it is not understood why breakthrough anti-D cases occur even when RhIg is administered properly.

We have previously described a murine model in which polyclonal anti-KEL (KELIg) immunoprophylaxis prevents alloimmunization to transfused RBCs expressing the human KEL glycoprotein when administered to recipient mice in their baseline state of heath (2). However, immunoprophylaxis fails in the setting of viral-like inflammation with poly (I:C) (3). We further showed that although type 1 interferon (IFN $\alpha / \beta)$ generation and sensing is sufficient to lead to immunoprophylaxis failure, it is not required (3). As such, the mechanism(s) leading to this breakthrough alloimmunization are only partially understood.

Complement plays a fundamental role in regulating immune responses, and we have previously reported that anti-KEL antibodies activate complement upon binding to KEL RBCs. Accordingly, we hypothesized that complement activation is involved in immunoprophylaxis failure and here we report that complement is required for immunoprophylaxis failure in the setting of poly (I:C). Complement receptors are likewise required, supporting a model of C3 opsonization of antibody bound RBCs that directly impacts immunoprophylaxis and its failure. These findings elucidate new and undescribed mechanistic details of antigen-antibody interactions.

\section{METHODS}

\section{Mice}

C57BL/6NCrl (B6) mice (strain code 027) were purchased from Charles River (Wilmington, MA). Complement C3 knockout, B6;129S4-C3/J(stock\#003641) (C3 $\left.{ }^{-/-}\right)$, C1qa knockout, B6(Cg)C1qatm1d(EUCOMM)Wtsi/TennJ (stock \#: $031675\left(\mathrm{Clq}^{-/-}\right.$), and CR1/2 knockout, B6.129S7(NOD)-Cr2tm1Hmo/J (Stock \#008225) (CR1/2/-) mice were purchased from Jackson (Bar Harbor, ME). Transgenic mice expressing the entire human KEL glycoprotein were previously generated; the mice used for these experiments have been previously described as "KEL2B" and express the KEL2 antigen in addition to the $\mathrm{Js}^{\mathrm{b}}$ antigen, the $\mathrm{Kp}^{\mathrm{b}}$ antigen, and other antigens in the KEL family on their RBCs (4). In this study they are referred to as "KEL" mice as the protein being studied includes the entire human KEL glycoprotein. All mice were housed in Yale University's animal facilities. All mice were 8-12 weeks of age and had a history of being backcrossed to the C57BL/6 background for at least 8 generations. All procedures and protocols were approved by Yale University's Institutional Care and Use Committee.

\section{KELIg Generation, Immunization, and Other Mouse Treatments}

Polyclonal KELIg antisera was generated as previously described (2), by transfusing transgenic murine KEL RBCs into B6 recipients pre-treated with an intraperitoneal injection of $100 \mu \mathrm{g}$ of high molecular weight poly (I:C) (Invivogen, San Diego, CA) a total of 3 times, separated by two weeks between each transfusion. Pooled sera collected 2-4 weeks after the final transfusion was tested for KEL binding ability by flow crossmatch with KEL or control B6 RBCs as targets; all IgG subtypes are represented (2). Following dose titration experiments, recipient mice were passively transferred with enough KELIg to lead to maximal RBC clearance (approximately 15 $\mu \mathrm{L}$ per experiment). In some experiments, poly (I:C) was administered approximately 3 hours prior to $\mathrm{RBC}$ transfusion.

\section{Blood Collection, Labeling, and Transfusion}

Donor KEL or wild type B6 RBCs were collected into anticoagulant preservative solution (CPDA, citrate phosphorus dextrose adenine, Jorgensen Labs, Henry Schein, Melville, NY), leukoreduced over a syringe filter (Pall Corporation, Port Washington, NY), and washed to remove residual citrate. Prior to transfusion in some experiments, B6 RBCs were labeled with chloromethylbenzamido 1,1'dioctadecyl-3,3,3',3'-tetramethylindocarbocyanine perchlorate (CM-DiI) and KEL RBCs were labeled with 3,3'-dihexad ecyloxacarbocyanine perchlorate (DiO) according to the manufacturer's instructions (Molecular Probes, Eugene, OR) and as previously described (5). Recipient mice were transfused via lateral tail with $50 \mu \mathrm{L}$ of KEL RBCs (in addition to control wild type RBCs in RBC recovery experiments). Survival of the transfused RBCs was determined by calculating the ratio of circulating $\mathrm{DiO}$ to DiI RBCs in recipients at select time points post-transfusion.

\section{Flow Cytometry}

Sera was collected at multiple time points and anti-KEL IgG responses were measured using a flow cytometric crossmatch assay with antigen positive (KEL) or antigen negative (B6) RBC targets. The secondary antibody was goat anti-mouse IgG (Jackson Immunoresearch, West Grove, PA). The antigen specific response [adjusted mean fluorescence intensity (MFI)] was determined by subtracting the signal of serum with antigen negative $\mathrm{B} 6 \mathrm{RBC}$ from that of serum with antigen positive RBCs. In experiments involving KELIg, the D0 timepoint was normalized to a fluorescence intensity of 1000 and a similar normalization was completed for the other timepoints. Transfused RBCs were analyzed for the KEL antigen by incubating with KELIg followed by anti-mouse IgG.

Following transfusion of KEL RBCs, complement was evaluated on the visualized RBC surface using antibodies against C3 (Cl7503B, Cedarlane, Ontario, Canada), C4 (clone 16D2, Santa Cruz), or Factor B (CL8824AP, Cedarlane, Ontario, Canada) followed by streptavidin or a fluorescently conjugated donkey anti-rabbit antibody (BioLegend, San Diego, California).

In other experiments, splenic cell subsets were evaluated following the transfusion of labeled RBCs. Spleens were harvested into ice-cooled RPMI 1640 media, finely minced 
with a razorblade, and processed into single-cell suspensions by passing the samples through a $70 \mu \mathrm{m}$ nylon cell strainer and collecting them on ice-cold FACS buffer (DPBS Modified, $0.2 \%$ BSA, 0.5 M EDTA). RBCs were lysed with ammonium chloride and splenocytes were treated with Fc block (anti-mouse CD16/ CD32; BD Biosciences, San Jose, CA) followed by incubation with cell surface antibodies. Antibodies against CD19 (clone 6D5), TCR $\beta$ (clone H57-597), CD11b (clone M1/70), CD11c (clone N418), CD8a (clone 53-6.7), F4/80 (clone BM8), CD115 (CSF-1R, clone AFS98), Ly-6G/Ly-6C (GR-1, clone RB6-8C5), and PDCA-1 (CD137, BST2, clone 927) were purchased from BioLegend (San Diego, CA). Anti-TER119 (clone TER119) was purchased from Invitrogen (Thermo Fisher Scientific Carlsbad, CA). Zombie Violet viability dye was purchased from BioLegend. For the invitro experiments involving peripheral blood WBCs, antibodies used also included CD19 (clone 6D5), B220 (clone RA3-6B2), CD21/35(clone 7E9), and CD23 (clone B3B4). Samples were analyzed on a BD LSR II cytometer, BD FACSCalibur, Miltenyi MACSQuant, or Beckman coulter CytoFlex S.

\section{In-Vitro Experiments}

In-vitro RBC experiments were completed by incubating KEL RBCs with KELIg, followed by the addition of serum, serum treated with EDTA, or serum treated with EGTA-Mg (Millipore Sigma); the serum was freshly collected from wild type mice and kept on ice until the addition of KEL RBCs. KELIg was diluted in GVB buffer with $\mathrm{Mg}$ and $\mathrm{Ca}$ (Complement Technology) and the cells were washed in GVB buffer without $\mathrm{Ca}$ or $\mathrm{Mg}$. Other in-vitro experiments involved incubating KEL RBCs (or wild type RBCs) with or without KELIg, following by incubation with sera or no sera, followed by incubation with peripheral blood WBCs from wild type mice or CR $1 / 2^{-/-}$mice, followed by staining for flow cytometric evaluation.

\section{Statistics and Images}

All statistical analysis was performed using Graph Pad Prism software (San Diego, CA). A Mann Whitney U test was used to determine significant differences between two groups, and ANOVA with Tukey's multiple comparisons test was completed in relevant experiments. Error bars represent one standard deviation, and significance was determined by a p-value less than 0.05. The visual abstract was created using BioRender.com.

\section{Data Sharing Statement}

For original data, please contact jeanne.hendrickson@yale.edu.

\section{RESULTS}

\section{Complement Is Fixed on KEL RBCs In-Vivo and In-Vitro in Response to Polyclonal Anti-KEL (KELIg)}

To evaluate the role of complement in immunoprophylaxis failure, we first tested whether passively transferred anti-KEL IgG (KELIg) leads to fixation by different complement components. Following KELIg infusion, KEL RBCs labeled with a lipophilic dye were transfused and RBCs recovered one hour later were evaluated for bound complement proteins (Figure 1A shows the experimental design). Complement C3, $\mathrm{C} 4$, and Factor $\mathrm{Bb}$ were detected bound to the recovered $\mathrm{DiO}$ labeled KEL RBCs in mice treated with KELIg, but not in control mice treated with saline (Figure 1B).

To determine which complement pathway was responsible, invitro experiments were completed. KEL RBCs were incubated with or without KELIg. After washing away unbound KELIg, KEL $\mathrm{RBCs}$ were incubated with serum from wild type mice and $\mathrm{RBC}$ bound complement C3, C4, and Factor Bb were measured. Similar to results from in-vivo experiments, $\mathrm{C} 3$ was readily detected bound to KEL RBCs in the condition involving KELIg and serum; C4 and $\mathrm{Bb}$ were more difficult to detect (Figure 1C). No complement was detected in KEL RBCs with serum or KEL RBCs with KELIg without serum control samples (Figure 1C). Other conditions included serum treated with EDTA (which blocks complement) or serum treated with EGTA and magnesium (which blocks the classical pathway of complement). Very little C3 could be detected under either of these conditions (Figure 1D), indicating the classical (antibody-mediated) complement pathway is most likely responsible for the RBC bound complement.

\section{Recipient Complement Is Required for KELIg Immunoprophylaxis Failure in Mice Treated With Poly (I:C)}

Since we observed that KELIg fixes complement (Figure 1), we tested the role of complement in the efficacy of immunoprophylaxis for recipients in their baseline state of health and following inflammation induced by poly (I:C). We found that immunoprophylaxis efficacy of KELIg when recipients are in their baseline state of health is not dependent on complement fixation, as KELIg remained as effective at preventing active anti-KEL formation in transfusion recipients lacking $\mathrm{C} 3$ as in wild type recipients (6) (Figures 2A, B). As shown in prior studies (2), the day 0 time point in Figure 2B and other figures involving KELIg is a measure of passive anti-KEL detected following KELIg administration; the day 14 time point represents a mixture of passively administered KELIg and actively formed anti-KEL, and by day 28 much of the passively administered KELIg is gone. However, in contrast to what we observed in wild type mice, poly (I:C) administration to $\mathrm{C}^{-1}$ recipients did not lead to immunoprophylaxis failure (Figure $2 \mathrm{C}$ ). This failure was likewise prevented in mice lacking C1q, which is upstream of C3 in the classical pathway of complement activation (Figure 2D) - suggesting that the classical pathway is required. Loss of poly (I:C) immunoprophylaxis failure was not due to an elimination of general poly (I:C) responsiveness in $\mathrm{C}^{-/-}$or $\mathrm{Clq}^{-/-}$ mice, as poly (I:C) induced a significant increase of anti-KEL IgG in response to KEL RBC transfusion in both strains (in the absence of pretreatment with KELIg) (Figure 2E).

\section{Recipient Complement Does Not Significantly Impact KEL RBC Clearance After KELlg in the Presence of Poly (I:C)}

Given that immunoprophylaxis failure occurred in wild type mice but not in $\mathrm{C}^{-/-}$or $\mathrm{Clq}^{-/-}$mice, we investigated the role of complement in clearance rates of transfused KEL RBCs. Following passive administration of KELIg and treatment with 

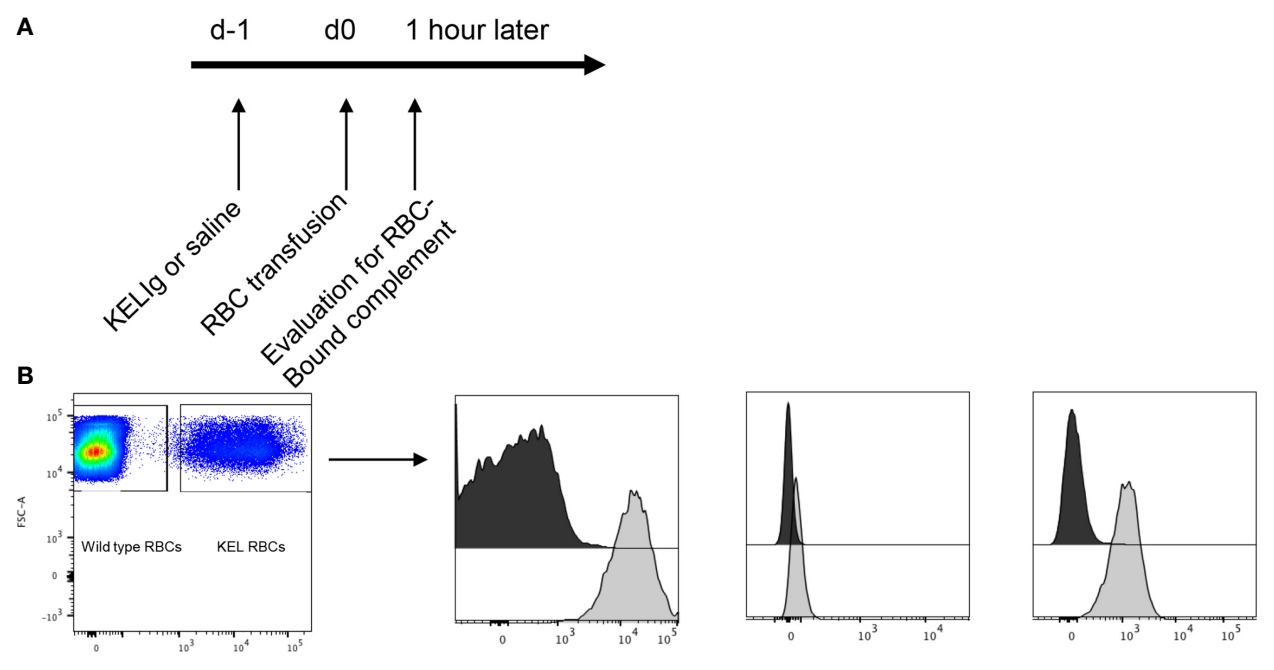

$\mathrm{DiO}$

C
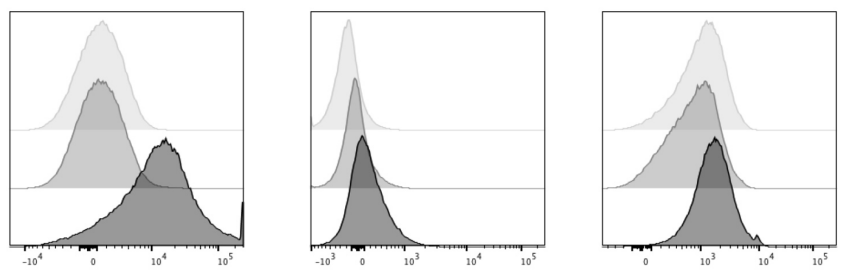

D
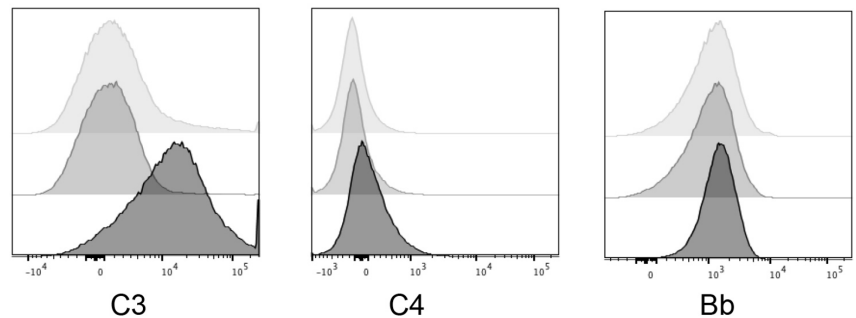

FIGURE 1 | Complement is fixed on KEL RBCs in-vivo and in-vitro in response to polyclonal anti-KEL (KELlg). (A) General in-vivo experimental design: recipients were treated with or without KELIg and transfused with DiO labeled KEL RBCs. (B) C3, C4, and Factor B were measured on recovered DiO positive KEL RBCs 1-hour post-transfusion; black filled histogram shows condition without KELIg; grey shaded histogram shows condition with KELIg. (C) In-vitro experiments were completed with KELIg incubated with KEL RBCs in the presence (darkest histogram) or absence (lightest histogram) of serum; an additional condition included saline incubated with KEL RBCs in the presence of serum (medium grey histogram). (D) Additional in-vitro conditions included KELIg incubated with KEL RBCs in the presence of serum (darkest histogram), in the presence of serum with EGTA-Mg (lightest histogram) or serum with EDTA (medium grey histogram). These data are representative of 3 independent experiments; $p<0.05$ for $\mathrm{C} 3$ detection on RBCs in the presence or absence of KELIg.

poly (I:C), recipient mice (wild type, $\mathrm{C}^{-{ }^{--}}$, and $\mathrm{C}^{-q^{--}}$) were transfused with DiO labeled KEL RBCs mixed with DiI labeled wild type RBCs. Blood was taken from recipient mice at 10 minutes, 1 hour, and 24 hours post-transfusion and the ratio of DiO KEL RBCs to DiI wild type RBCs was evaluated. As we have observed when KELIg is administered in the absence of inflammation (6), $\mathrm{C}^{-1-}$ mice had slightly delayed RBC clearance compared with wild type mice in the presence of inflammation (Figure 3A), with $\mathrm{Clq}^{-1-}$ mice having similar clearance patterns compared with wild type mice (Figure 3B); Supplemental Figure 1 shows $\mathrm{C}^{-1-}$ and $\mathrm{Clq}^{-1-}$ recipient $\mathrm{RBC}$ clearance following KELIg in the absence of poly (I:C).

The recovered DiO labeled KEL RBCs were then evaluated for the presence of the KEL glycoprotein antigen by incubating the recovered cells with anti-KEL antibody followed by fluorescently labeled mouse IgG. As previously shown, KEL antigen expression on RBCs remains stable at 10 minutes, 1 hour, and 24 hours posttransfusion in wild type mice transfused in the absence of KELIg (2), and antigen expression is similar in the presence or absence of poly (I:C) (3). The absence of C3 (Figure 3C), but not C1q (Figure 3D), delayed KEL glycoprotein antigen modulation compared with wild type mice after treatment with KELIg in the presence of poly (I:C), for reasons that are not entirely self-evident. Taken in combination, however, it is unlikely that either RBC clearance or antigen modulation are responsible for explaining the differences in immunoprophylaxis efficacy observed between complement deficient and wild type mice in the setting of inflammation. 
A

B

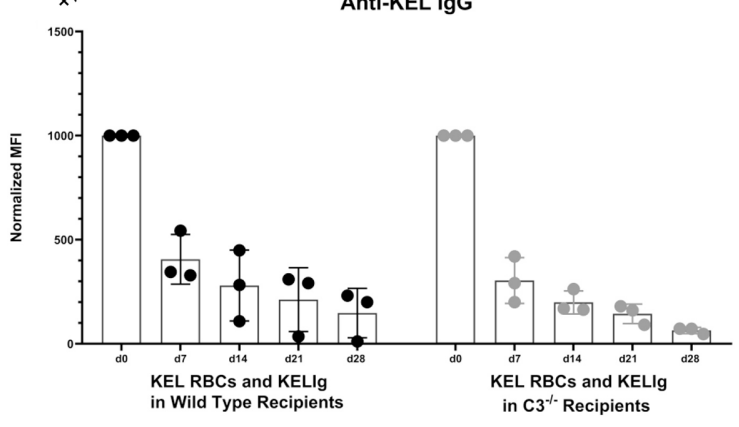

D

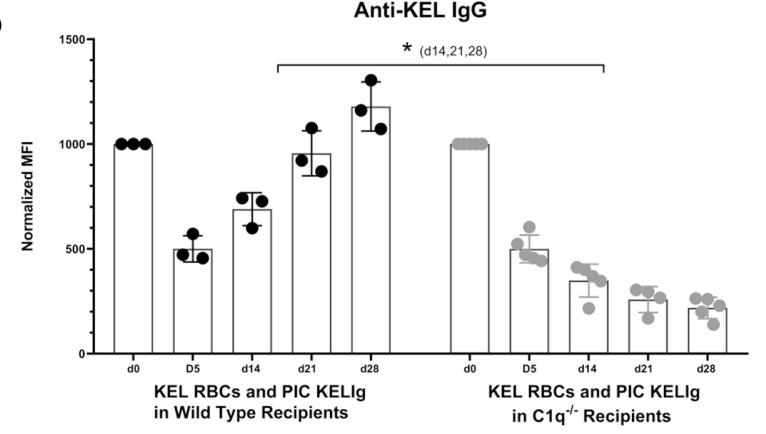

c

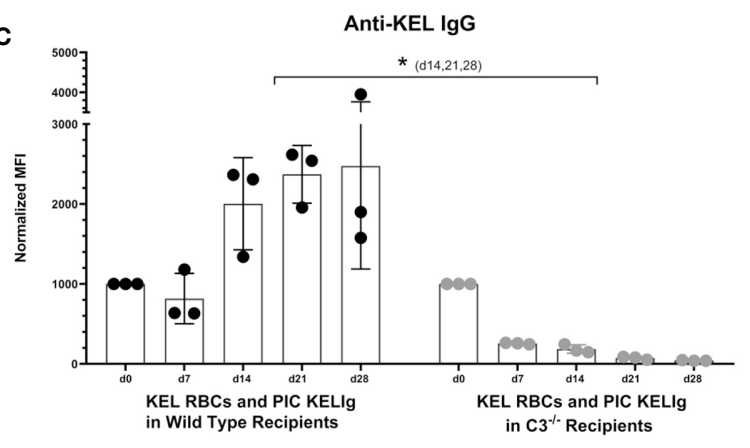

$E$

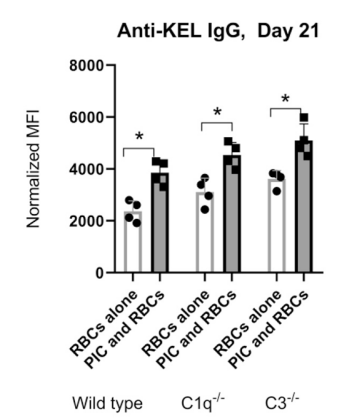

FIGURE 2 | Recipient C3 and C1q contribute to KELlg immunoprophylaxis failure in the setting of poly (I:C). (A) General in-vivo experimental design: recipients were infused with KELlg and transfused the following day in the absence or presence of poly (I:C) treatment, with anti-KEL responses evaluated longitudinally. (B) Anti-KEL IgG responses in wild type compared with $\mathrm{C}^{-/}$recipients transfused in the absence of poly (I:C). (C) Anti-KEL IgG responses in wild type compared with $\mathrm{C}^{-/-}$ recipients transfused in the presence of poly (I:C). (D) Anti-KEL IgG responses in wild type compared with $\mathrm{C}^{-\mathrm{q}^{-/-}}$recipients transfused in the presence of poly (I:C). (E) Baseline impact of recipient treatment with poly (I:C) on alloimmune responses to KEL RBCs in the absence of KELIg. These data are representative of 2-3 independent experiments, with 3-5 mice/group/experiment; error bars indicate standard deviation between individual mice. ${ }^{*} p<0.05$ (C, D) d14,21,28, and all comparisons for (E).

\section{Recipient Complement Enhances KEL RBC Uptake by Inflammatory Monocytes After KELlg in the Presence of Poly (I:C)}

Complement is known to mediate antigen uptake by antigen presenting cells (APCs) (7). Thus, despite the relatively similar overall peripheral $\mathrm{RBC}$ clearance patterns in wild type mice compared with those lacking complement, we hypothesized that the incompatible KEL RBCs may be consumed by different APCs more likely to promote alloantibody formation or that the APCs consuming the transfused RBCs may be differentially activated in the presence of complement. To evaluate RBC consumption, wild type and $\mathrm{C}^{-1-}$ mice were transfused with $\mathrm{DiO}$ labeled KEL RBCs and leukocyte subsets in their spleens were evaluated 1 hour and 16 hours later. First, we gated on single cells, live cells, and non-T/non-B/non-RBCs (Supplemental Figure 2). Next, splenic red pulp macrophages, dendritic cells $(\mathrm{CD} 8 \alpha, \mathrm{CD} 11 \mathrm{~b}$ and plasmacytoid), resident and inflammatory monocytes, and neutrophils were quantified by percentage one hour (Figure 4A) and 16 hours (Figure $\mathbf{4 B}$ ) post-transfusion. Differences in these cell subsets were observed between genotypes as shown, with an increase in neutrophils after poly (I:C) treatment in wild type mice being particularly prominent by 16 hours post-transfusion. Next, RBC consumption of transfused DiO labeled KEL RBCs by each of these cell subsets was determined. One difference between wild type and $\mathrm{C}^{-/-}$mice following KELIg and poly (I:C), compared with KELIg alone, was the increase in $\mathrm{DiO}$ labeled KEL RBC consumption by inflammatory monocytes that occurred in wild type mice and that was seen to a lesser degree in $\mathrm{C}^{-1-}$ mice (Figure 4C shows a histogram example at 16 hours post-transfusion); these changes were evident one hour posttransfusion (Figure 4D) but were even more pronounced by 16 hours post-transfusion (Figure 4E). 
A

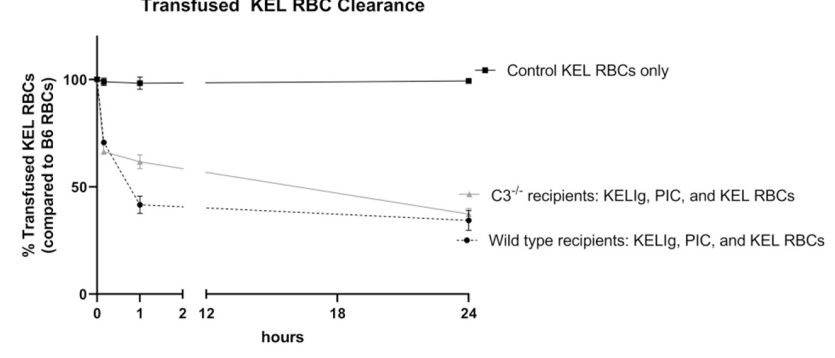

C

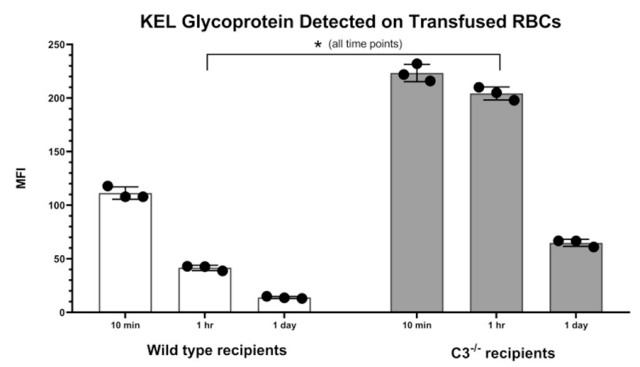

B

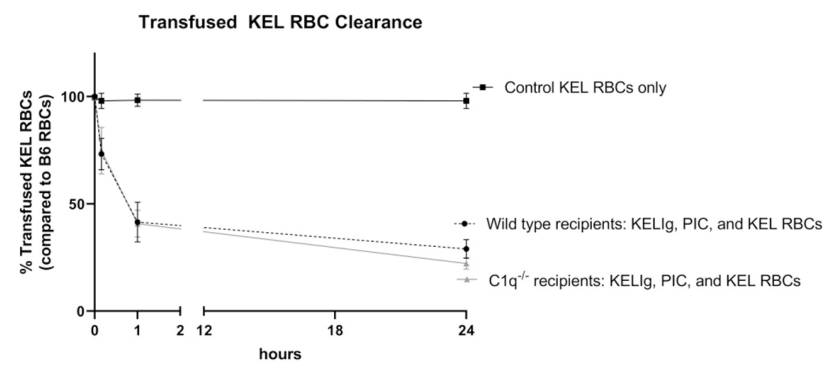

D

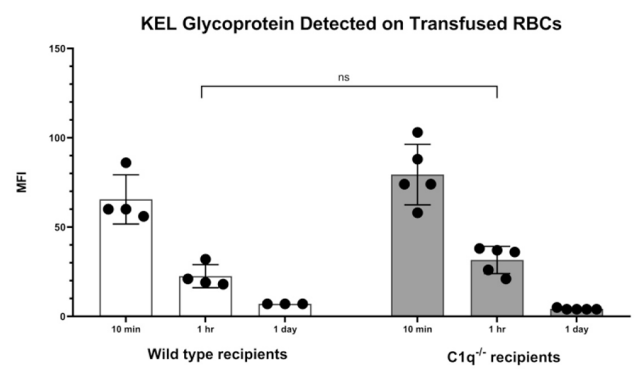

FIGURE 3 | Recipient complement, KEL RBC clearance, and KEL antigen expression. KEL RBCs were labeled with DiO and mixed with wild type RBCs labeled with Dil; this mixture was transfused into recipients that had been infused with KELIg and treated with poly (I:C). (A) shows wild type compared with C3 ${ }^{-/-}$recipients; (B) shows wild type compared with $\mathrm{C}_{1} \mathrm{q}^{-/-}$recipients. Recovered DiO labeled RBCs were then evaluated for KEL glycoprotein expression by flow cytometry, after incubation with KELIg and fluorescently conjugated anti-mouse IgG; (C) shows wild type recipients compared with C3 $^{-/-}$recipients; (D) shows wild type recipients

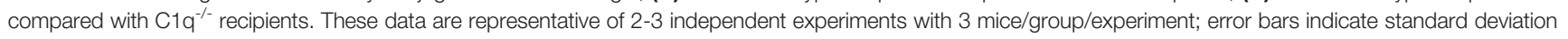
between mice. ${ }^{*} p<0.05$ for all comparisons in (C) and $p=n s$, not significant for all comparisons in (D).

In addition, monocytes and neutrophils were evaluated in the peripheral blood at the 1 hour and 16-hour post-transfusion time points (Supplemental Figures 3A, B). Similar to trends observed in the spleen, peripheral blood inflammatory monocytes in the wild type mice had significantly more DiO signal inside them compared with those in the $\mathrm{C}^{-1-}$ mice (Supplemental Figures 3C, D). Taken in combination, these findings suggest that in the setting of inflammation, complement fixation may direct the KEL antigen-containing RBCs towards antigen presenting cells more likely to ultimately contribute to a productive antibody response.

\section{Complement-Fixed KEL RBCs Bind In-Vitro to B-Cells From Wild Type Mice}

As the interaction of complement, antigen, and complement receptors has long been known to be critical for regulating antibody production (8), we next turned our attention to complement receptors on B-cells. Work by others has shown that complement receptors 1 and $2(\mathrm{CR} 1 / 2)$ are present on follicular dendritic cells and B-cells in mice, and crosslinking of the B-cell receptor (BCR) and $\mathrm{CR} 1 / 2$ on $\mathrm{B}$-cells lowers the activation threshold (9). As a first step to evaluate the role of complement binding to B-cells in our model, we incubated DiO labeled KEL or wild type RBCs with or without KELIg in-vitro. After washing away unbound KELIg, the RBCs were incubated with sera and white blood cells (WBCs). CD19+B220+ WBCs that were positive for $\mathrm{DiO}$ had a mixture of $\mathrm{C} 3$ positivity and $\mathrm{C} 3$ negativity under conditions including KEL RBCs + KELIg + sera, and CD19+ $\mathrm{B} 220+$ WBCs that were negative for $\mathrm{DiO}$ were $\mathrm{C} 3$ negative (Figure 5A). Control conditions, including those lacking KEL RBCs, those lacking KELIg, and those lacking sera showed no C3 positivity (Supplemental Figure 4). To investigate which CD19 + B220+ WBCs bound the complement fixed KEL RBCs, we utilized CD23 and CD21/35 staining. The CD19+B220+ cells most highly positive for CD23 and CD21/35 (largest gate) that were positive for $\mathrm{DiO}$ had uniform C3 positivity, whereas the CD19+B220+ cells with less strong CD23 and CD21/35 positivity (smallest gate) that were positive for $\mathrm{DiO}$ were predominantly $\mathrm{C} 3$ negative. The conditions were then expanded, comparing WBCs from $\mathrm{CR} 1 / 2^{-/-}$ mice with those from wild type mice. The CD19+B220+ B-cells from $\mathrm{CR} 1 / 2^{-/-}$mice showed decreased binding to KEL RBCs compared with B-cells from wild type mice. Further, the CR1/2-/B-cells that bound DiO labeled KEL RBCs had less detectable C3 compared with those from wild type mice (Figure 5B). These results suggest that B-cells bind to opsonized, complement fixed KEL RBCs through CR1/2, raising a question of the role these receptors play in immunoprophylaxis failure.

\section{Complement Receptors (CR1/2) Are Necessary for KELIg Immunoprophylaxis Failure in the Presence of Poly (I:C)}

To evaluate the functional role of CR1/2 in the immunoprophylaxis setting of KELIg, we first needed to establish that these mice could generate an anti-KEL response following a KEL RBC transfusion. 
A

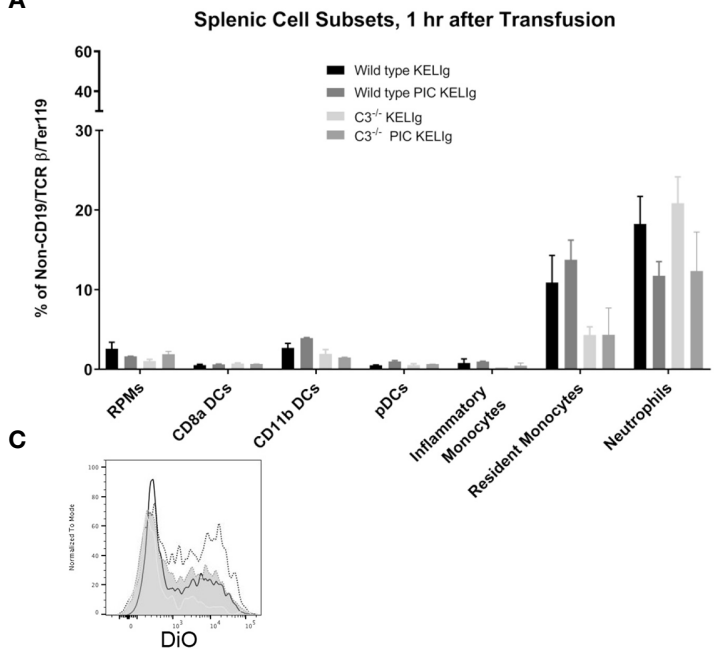

D

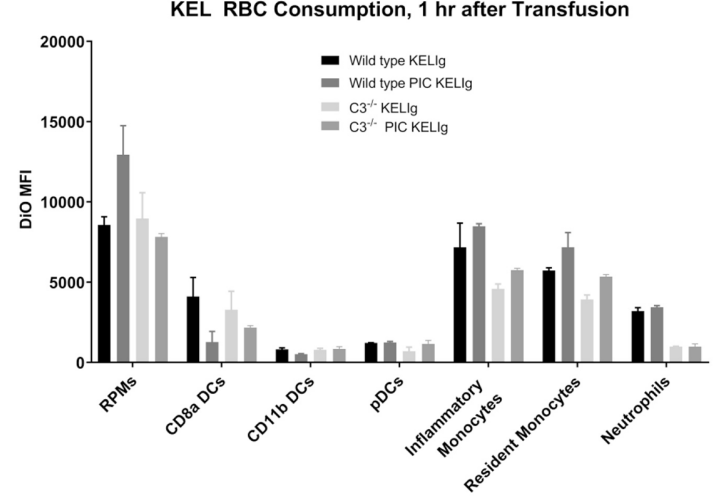

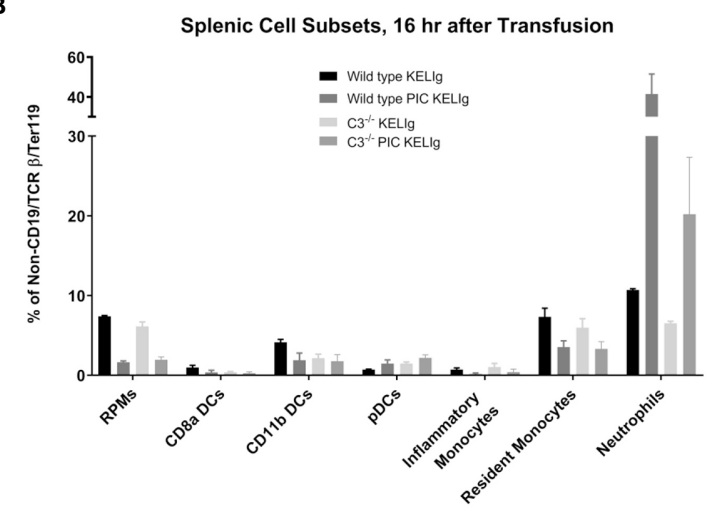

E

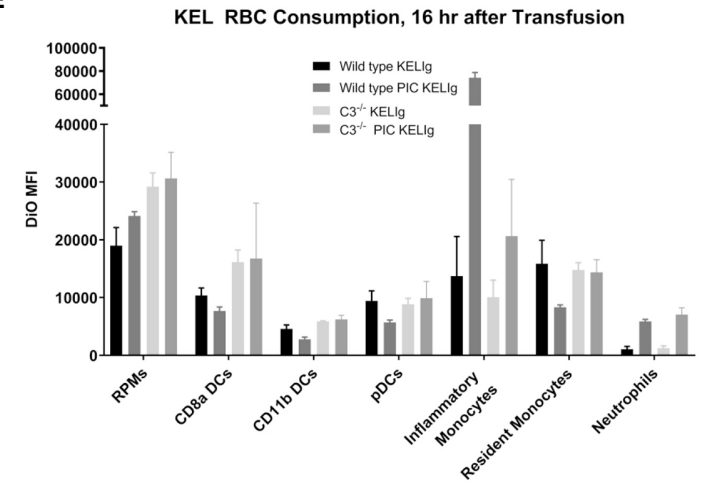

FIGURE 4 | Poly (l:C) and KELlg immunoprophylaxis result in increased transfused KEL RBC consumption by splenic inflammatory monocytes in wild type compared with $\mathrm{C}^{-1-}$ mice. DiO labeled KEL RBCs were transfused to wild type or $\mathrm{C}^{-1}$ mice treated with KELlg in the presence or absence of poly $(I: \mathrm{C})$ and splenic cell subsets were evaluated at 1 and 16 hours post-transfusion (A, B). (C) Representative histograms for DiO RBC fluorescence patterns of inflammatory monocytes at 16 hours posttransfusion, after first excluding TER119 positive RBCs on the exterior of the splenic cells; black open histogram is KELlg in wild type, dotted open histogram is KELlg and $\mathrm{PIC}$ in wild type; white shaded histogram is $\mathrm{KELL}$ in $\mathrm{C}^{-1}$ and dotted shaded histogram is KELlg and PIC in $\mathrm{C}^{-1}$. DiO mean fluorescence intensity (MFI) of the splenic cell subsets was evaluated at 1 hour (D) and 16 hours (E) post-transfusion. These data are representative of 2-3 independent experiments with 3 mice/group/experiment; error bars indicate standard deviation between mice.

Similar to wild type mice, we observed that $\mathrm{CR} 1 / 2^{-/-}$recipients were able to generate anti-KEL IgG antibodies following a KEL RBC transfusion and to generate an enhanced response in the presence of poly (I:C), albeit lower in magnitude than that observed in wild type mice (Figure 6A). Next, we evaluated the response of CR $1 / 2^{-/-}$mice to KEL RBCs after prophylaxis with KELIg. As we have observed in wild type mice, KELIg prevented anti-KEL alloantibody responses in $\mathrm{CR} 1 / 2^{-/-}$mice (data not shown). Compared to wild type mice, the KEL RBCs recovered from the CR1/2/- mice had similar posttransfusion recover and survival as wild type recipients (Figure 6B), with delayed kinetics of KEL glycoprotein antigen modulation (Figure 6C) and a trend towards lower levels of bound C3 (Figure 6D). However, in the setting of poly (I:C)-induced inflammation and KELIg, immunoprophylaxis failure and breakthrough alloimmunization did not occur in $\mathrm{CR} 1 / 2^{-/-}$mice (Figure 6E). Taken together, these data indicate that CR1/2 are required for immunoprophylaxis failure and further emphasize the lack of an association between peripheral RBC clearance rates and outcome, supporting a model in which complement functions in breakthrough alloimmunization by promoting antibody-generating immune responses.

\section{DISCUSSION}

These studies were undertaken to better understand why polyclonal anti-KEL IgG (KELIg) prevents alloimmunization when a recipient is exposed to KEL RBCs in a baseline state of health but fails to prevent alloimmunization (e.g. immunoprophylaxis failure) when the recipient is in a state of viral-like inflammation. Prior studies suggested that type 1 IFN (IFN- $\alpha / \beta)$ generated in recipients after poly (I:C) treatment was sufficient but not necessary to lead to immunoprophylaxis failure (3), leading us to search for other pathways. The take home point of the current studies is that 


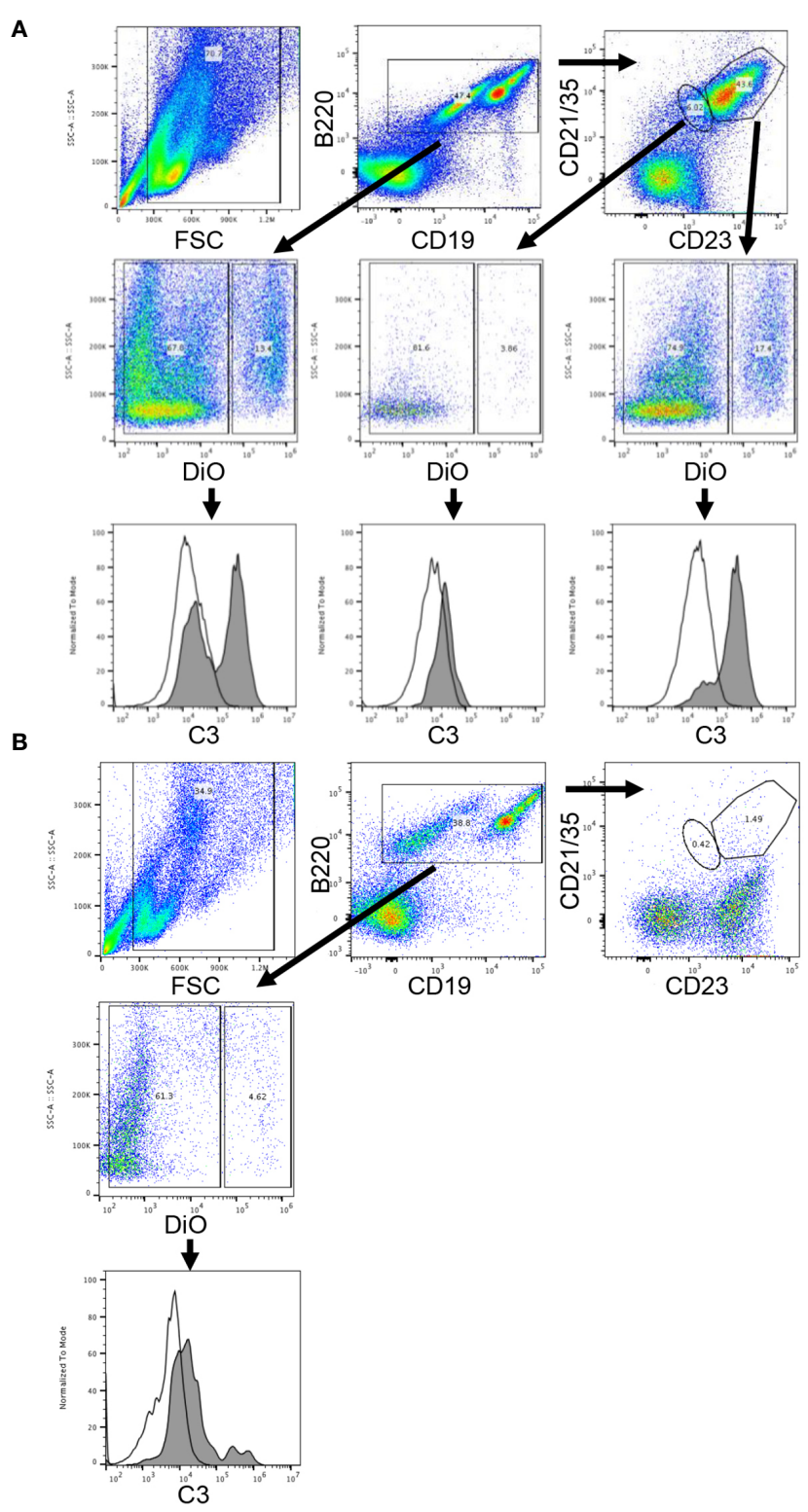

FIGURE 5 | Complement-fixed KEL RBCs bind in-vitro to wild type donor derived B-cells but not to CR1/2/- B-cells. DiO labeled KEL RBCs were incubated with KELIg in the presence of sera, followed by incubation with peripheral blood derived WBCs from donor mice. (A) In wells using WBCs from wild type donor mice, CD19+B220+B-cells cells were separated by DiO positivity and then by C3 positivity. The CD19+B220+ cells were next separated by their CD23 and CD21/35 expression, with the cells highest for CD23 and CD21/35 gated in the larger gate and those less strongly positive for CD23 and CD21/35 gated in the smaller gate; the DiO positive and negative populations were then evaluated for their C3 positivity. Shaded histograms are the DiO positive population, open histograms are the DiO negative population. (B) In wells using WBCs from CR1/2/- mice, CD19+B220+ B-cells cells were separated by DiO positivity and then by C3 positivity. Shaded histograms are the DiO positive population, open histograms are the DiO negative population. These data are representative of more than 3 independent experiments, with 2 involving CD23 and CD21/35 staining. recipient complement is a key mediator in inflammation-associated immunoprophylaxis failure. As such, we propose a "two-hit" hypothesis, where the first "hit" is recipient inflammation, and the second "hit" is complement production/sensing (Figure 7).

Our findings thus support a model in which immunoprophylaxis fails in the setting of inflammation due to complement-mediated promotion of alloantibody generation. The classical complement pathway is activated by the binding of KELIg to KEL RBCs and the alternative complement pathway is activated by poly (I:C) (10-12). Free heme, generated as a result of RBC breakdown and known to activate the alternative complement pathway (13), may also be playing a role. Once generated, activated complement binds to complement receptors (CR1/2) on B-cells to lower the activation threshold for antibody generation. The requirement of $\mathrm{C} 3$ or $\mathrm{C} 1 \mathrm{q}$ for immunoprophylaxis failure support this model. Further, the requirement of CR1/2 for immunoprophylaxis failure and the decreased binding of complement coated opsonized RBCs in-vitro to $\mathrm{CR} 1 / 2^{-/-}$B-cells lead us to suspect that B-cells play a critical "gatekeeper" role in our model.

$\mathrm{CR} 1 / 2$ on B-cells have been described to function as type 1 IFN (IFN- $\alpha / \beta$ ) receptors (14), something of interest given our past studies showing that type 1 IFN is sufficient but not necessary for inflammation-associated immunoprophylaxis failure. It is likely that consumption of antibody coated RBCs by inflammatory monocytes, potentially driven by type 1 IFN production or the anaphylatoxins $\mathrm{C} 3 \mathrm{a}$ and $\mathrm{C} 5 \mathrm{a}$ [and presumably resulting in the generation of cytokines including type 1 IFN as well as C3a and C5a which are further known to activate antigen presenting cell/T-cell interactions and to create feedback loops $(15,16)]$, contribute in part to our findings. In addition to serving as type 1 IFN receptors, CR1/2 are key markers of marginal zone B cells that distinguish them from follicular B cells; past studies in the KEL system have shown the necessity of marginal zone B-cells but not follicular Bcells for anti-KEL IgG responses (17). Recent studies suggest that marginal zone B cell-mediated RBC alloimmunization may not require transport of RBCs to the splenic follicle (18), pointing to an intrinsic role of CR1/2 in B cell signaling following engagement of $\mathrm{RBC}$ bearing both alloantigen and $\mathrm{C} 3$ split products. While prior studies demonstrated the importance of CR1/2 on B cells in antiKEL IgG responses in the absence poly (I:C) (19), we cannot exclude the relevance of follicular dendritic cells to our present findings. Of note, models of systemic lupus erythematosus have shown the uptake of complement associated antigen by follicular DCs generates type 1 IFN in an IRF-5 dependent pathway (20).

Past studies have taught us that recipient complement and $\mathrm{Fc} \gamma$ receptors in combination are important in incompatible $\mathrm{RBC}$ clearance, KEL antigen modulation, and immunoprophylaxis efficacy when a recipient is in a baseline state of health (6). Although we previously thought RBC clearance patterns might impact immunoprophylaxis efficacy, recent studies (3) (including the present studies) show that may not be true in all settings. The data presented here support observations by Stegmann (21) and others (22) that RBC clearance patterns alone may be inadequate to fully evaluate the immunoprophylaxis efficacy of candidate monoclonal or polyclonal antibodies. 
A

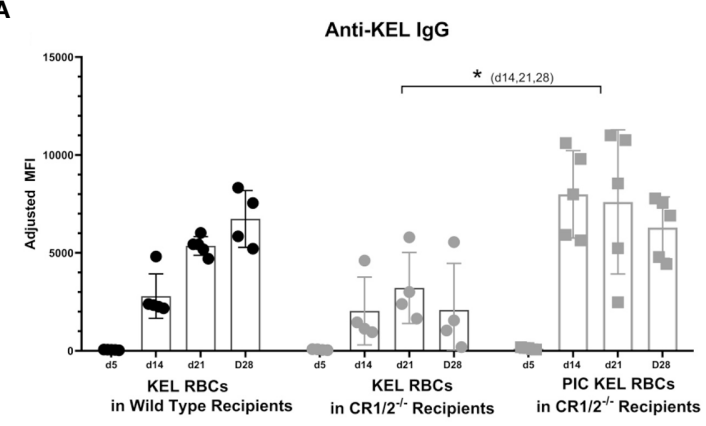

C

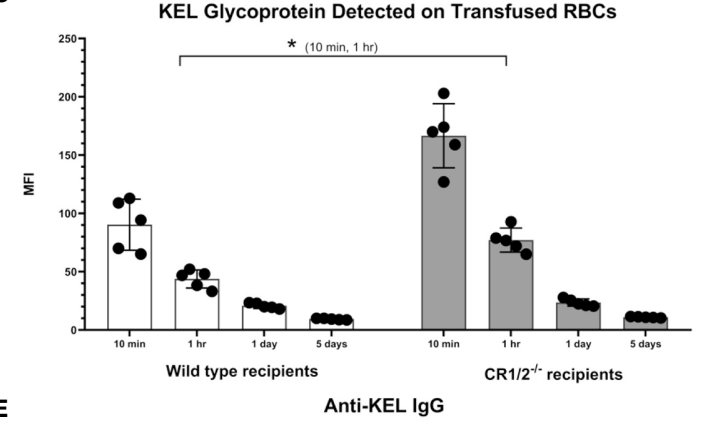

E

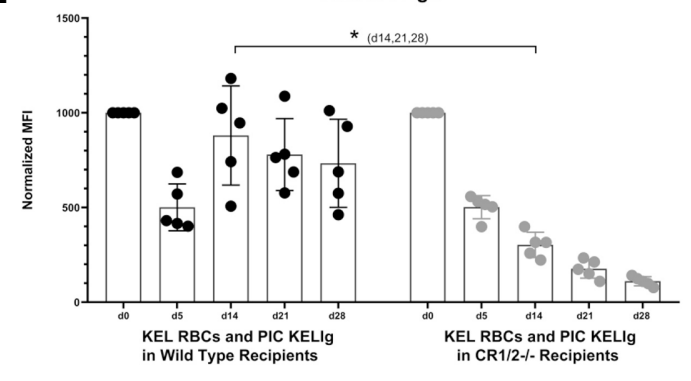

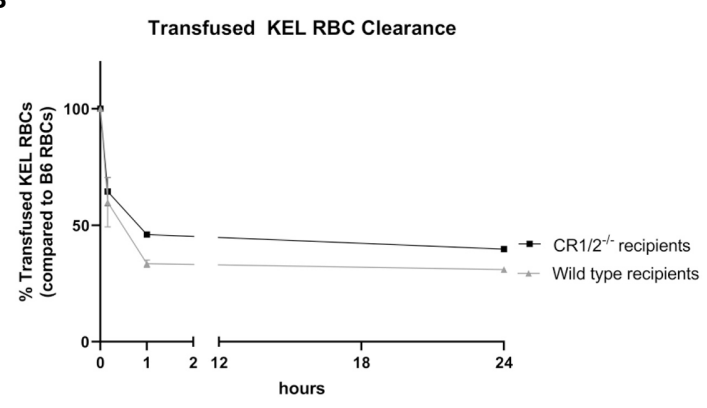

D

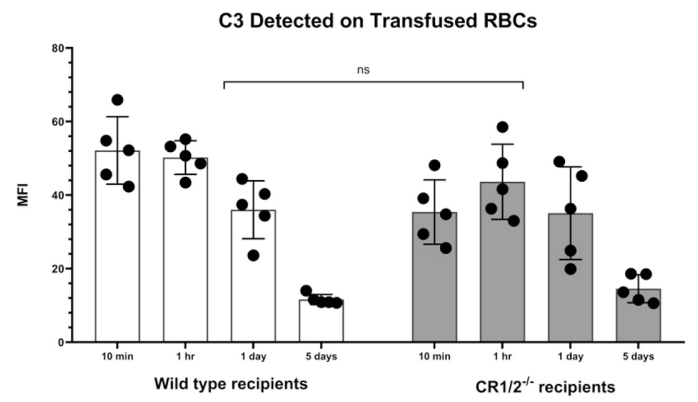

FIGURE 6 | CR1/2 are required for KELIg immunoprophylaxis failure in the setting of poly (l:C). (A) KEL RBCs were transfused into wild type recipients or recipients lacking CR1/2, in the presence or absence of poly (l:C). (B) RBCs were labeled with DiO and mixed with wild type RBCs labeled with Dil; this mixture was transfused into wild type or CR1/2/- recipients that had been infused with KELlg and treated with poly (l:C). Recovered DiO positive KEL RBCs were evaluated for (C) KEL glycoprotein expression, and (D) bound complement C3. (E) Wild type or CR1/2/- recipients were infused with KELlg and transfused the following day in the absence or presence of poly (l:C), with anti-KEL responses evaluated longitudinally. ${ }^{*} \mathrm{p}<0.05$ for $\mathrm{d} 14,21$ and 28 of $(\mathbf{A})$ between $\mathrm{CR} 1 / 2^{-/-}$mice treated with or without poly (I:C); $\mathrm{p}<0.05$ for 10 min and 1-hour timepoints in (C); $p<0.05$ for d14, 21, and 28 in (E). These data are representative of 2-3 independent experiments with 3 mice/group/experiment; error bars indicate standard deviation between mice. ns, not significant.

Our model and findings may have translational relevance for better understanding immunoprophylaxis failures (to RhIg). It remains unclear why some women become alloimmunized against the $\mathrm{D}$ antigen during pregnancy/childbirth despite prophylaxis with polyclonal RhIg $(21,23)$. FcyR 2/3 polymorphisms have been evaluated in such women, with the conclusion that highaffinity alleles encoding these receptors do not influence RhIg efficacy. Although antibodies to the RhD antigen are thought not thought to fix complement on RBCs themselves, it is possible that background complement activation or $\mathrm{C} 3 \mathrm{a} / \mathrm{C} 5 \mathrm{a}$ generation as a result of heme or inflammation-associated complement pathway activation may play a role. Increasing evidence also supports the role of complement activation (24) in the pathophysiology of antibody positive as well as antibody negative (25) delayed hemolytic transfusion reactions, with complement blockade being shown to mitigate lifethreatening reactions (26).

Study limitations should be considered. These studies were focused on a single blood group antigen and a single type of inflammation. Another consideration is that responses to different antigens on murine RBCs involve different pathways, with some being T-cell dependent (27) and others being T-cell independent; this is presumably true in humans as well. Complement has recently been shown to serve as a "switch" between CD4+ T-cell dependent and independent responses (19) and it is possible that poly (I:C) induced immunoprophylaxis failure is overcome by the CD4+ T-cell dependency of the alloantibody response in complement deficient mice. Our KELIg preparation is polyclonal with all IgG subtypes represented; monoclonal antibodies, either individually or in combination, may give different results (28). 


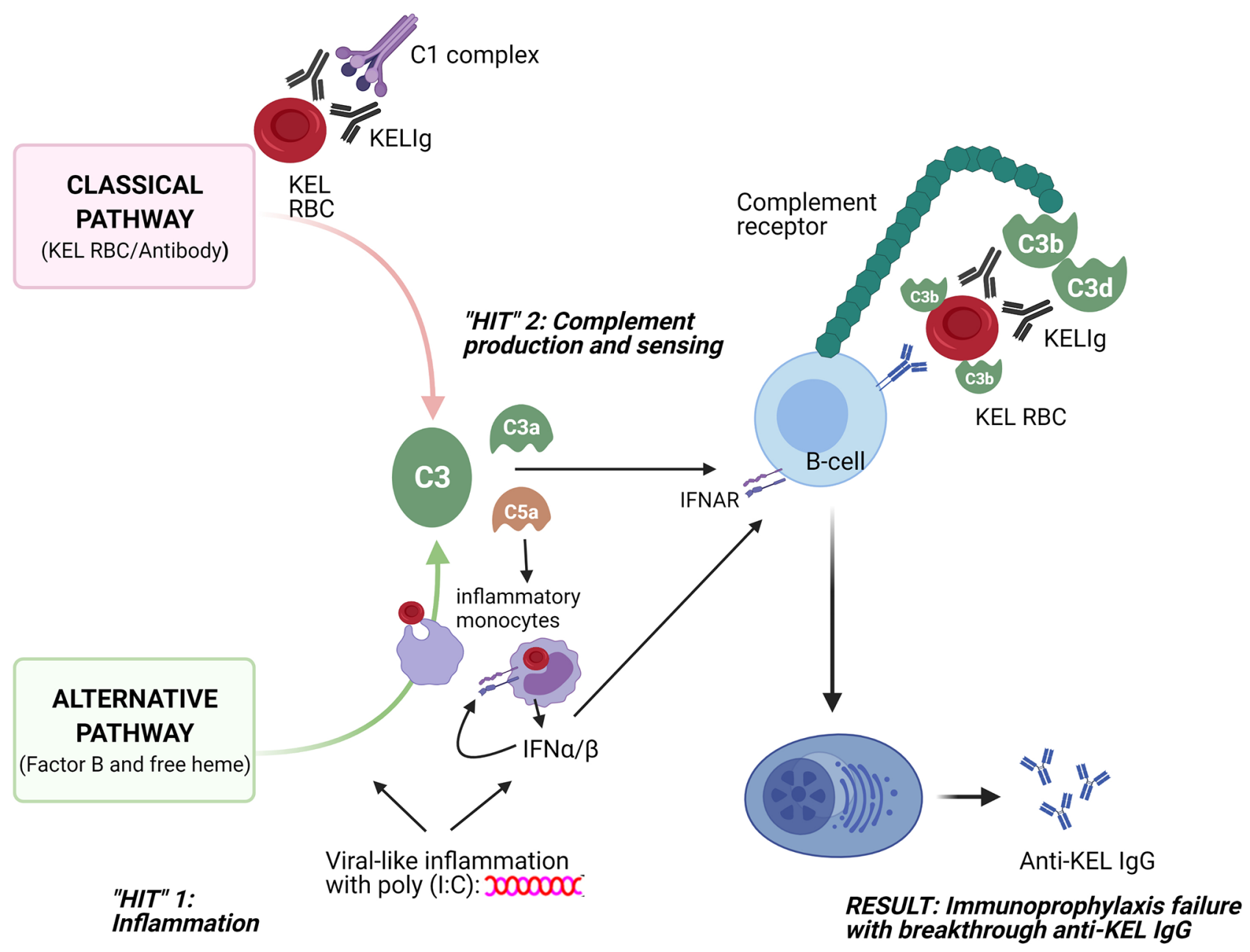

FIGURE 7 | Schematic of 2 "hits" resulting in immunoprophylaxis failure in the setting of viral-like inflammation. The first "hit" involves recipient inflammation with poly $(I: C)$, with resultant type $1(\alpha / \beta)$ interferon production among other things; the second "hit" involves complement generation through the classical pathway due to antibody/antigen interactions and through the alternative pathway due to poly $(\mathrm{l}: \mathrm{C})$ and potentially due to free heme generation. Consumption of opsonized RBCs by inflammatory monocytes likely also plays a key role, with type 1 IFN and C3a and C5a potentially increasing this consumption and with type 1 IFN generation likely having an impact on downstream B-cells. The sum of these two "hits" is that opsonized KEL RBCs with fixed complement bind to CR1/2 on B-cells, lowering the activation threshold for anti-KEL formation and resulting in inflammation-induced immunoprophylaxis failure.

Further, in contrast to KEL, immunoprophylaxis to murine RBCs expressing an alternate (HOD, containing HEL) antigen can occur independent of complement or Fc $\gamma$ receptors, suggesting that distinct antigen-antibody combinations may dictate the relative influence of complement, Fc $\gamma$ receptors and inflammation on the success or failure of immunoprophylaxis (29-31). Indeed, antiKEL and anti-HEL antibodies can induce antigen specific immunoprophylaxis following exposure to RBCs expressing both the KEL and HOD antigens (32). In contrast, antibodies directed toward one antigen may enhance or inhibit alloantibody formation against the non-target alloantigen (32-34). As such, distinct mechanisms of immunoprophylaxis should be considered depending on the target alloantigen involved $(35,36)$. One final consideration is that co-engagement of the $\mathrm{B}$-cell receptor and complement receptors in humans may lead to different responses than those observed in mice (37).
In conclusion, our data highlight the critical role that complement plays in inflammation-induced immunoprophylaxis failure. Generated in one model, the mechanisms described here likely have broader applicability. Although complement was identified as contributing to disease more than half a century ago (38), it is likely that the era of studying and targeting complement activation and break down products, in transfusion medicine and beyond, has just begun.

\section{DATA AVAILABILITY STATEMENT}

The original contributions presented in the study are included in the article/Supplementary Material, further inquiries can be directed to the corresponding author. 


\section{ETHICS STATEMENT}

The animal study was reviewed and approved by Yale University IACUC.

\section{AUTHOR CONTRIBUTIONS}

VE-R, MS, JL, and JH planned the experiments. VE-R, MS, and JL executed most of the experiments. All authors made experimental suggestions and interpreted experimental results. $\mathrm{JH}$ wrote the initial draft of the manuscript, and all authors edited the manuscript and approved the final version of the manuscript.

\section{REFERENCES}

1. Vossoughi S, Spitalnik SL. Conquering Erythroblastosis Fetalis: 50 Years of Rhig. Transfusion (2019) 59(7):2195-6. doi: 10.1111/trf.15307

2. Stowell SR, Arthur CM, Girard-Pierce KR, Sullivan HC, Santhanakrishnan M, Natarajan P, et al. Anti-KEL Sera Prevents Alloimmunization to Transfused KEL Rbcs in a Murine Model. Haematologica (2015) 100(10):e394-7. doi: 10.3324/haematol.2015.128603

3. Escamilla-Rivera V, Liu J, Gibb DR, Santhanakrishnan M, Liu D, Forsmo JE, et al. Poly(I:C) Causes Failure of Immunoprophylaxis to Red Blood Cells Expressing the KEL Glycoprotein in Mice. Blood (2020) 135(22):1983-93. doi: 10.1182/blood.2020005018

4. Smith NH, Henry KL, Cadwell CM, Bennett A, Hendrickson JE, Frame T, et al. Generation of Transgenic Mice With Antithetical KEL1 and KEL2 Human Blood Group Antigens on Red Blood Cells. Transfusion (2012) 52 (12):2620-30. doi: 10.1111/j.1537-2995.2012.03641.x

5. Girard-Pierce KR, Stowell SR, Smith NH, Arthur CM, Sullivan HC, Hendrickson JE, et al. A Novel Role for C3 in Antibody-Induced Red Blood Cell Clearance and Antigen Modulation. Blood (2013) 122(10):1793801. doi: 10.1182/blood-2013-06-508952

6. Liu J, Santhanakrishnan M, Natarajan P, Gibb DR, Eisenbarth SC, Tormey CA, et al. Antigen Modulation as a Potential Mechanism of Anti-KEL Immunoprophylaxis in Mice. Blood (2016) 128(26):3159-68. doi: 10.1182/blood-2016-06-724732

7. Jacquier-Sarlin MR, Gabert FM, Villiers MB, Colomb MG. Modulation of Antigen Processing and Presentation by Covalently Linked Complement C3b Fragment. Immunology (1995) 84(1):164-70.

8. Carroll MC, Isenman DE. Regulation of Humoral Immunity by Complement. Immunity (2012) 37(2):199-207. doi: 10.1016/j.immuni.2012.08.002

9. Carter RH, Spycher MO, Ng YC, Hoffman R, Fearon DT. Synergistic Interaction Between Complement Receptor Type 2 and Membrane IgM on B Lymphocytes. J Immunol (1988) 141(2):457-63.

10. Zhang X, Kimura Y, Fang C, Zhou L, Sfyroera G, Lambris JD, et al. Regulation of Toll-like Receptor-Mediated Inflammatory Response by Complement In Vivo. Blood (2007) 110(1):228-36. doi: 10.1182/blood-2006-12-063636

11. Kaczorowski DJ, Afrazi A, Scott MJ, Kwak JH, Gill R, Edmonds RD, et al. Pivotal Advance: The Pattern Recognition Receptor Ligands Lipopolysaccharide and Polyinosine-Polycytidylic Acid Stimulate Factor B Synthesis by the Macrophage Through Distinct But Overlapping Mechanisms. J Leukoc Biol (2010) 88(4):609-18. doi: 10.1189/jlb.0809588

12. Zou L, Feng Y, Xu G, Jian W, Chao W. Splenic RNA and MicroRNA Mimics Promote Complement Factor B Production and Alternative Pathway Activation Via Innate Immune Signaling. J Immunol (2016) 196(6):278898. doi: 10.4049/jimmunol.1502106

13. Thomas AM, Gerogianni A, McAdam MB, Fløisand Y, Lau C, Espevik T, et al. Complement Component C5 and TLR Molecule CD14 Mediate HemeInduced Thromboinflammation in Human Blood. J Immunol (2019) 203 (6):1571-8. doi: 10.4049/jimmunol.1900047

14. Kim D, Niewiesk S. Synergistic Induction of Interferon $\alpha$ Through TLR-3 and TLR-9 Agonists Identifies CD21 as Interferon $\alpha$ Receptor for the B Cell Response. PLoS Pathog (2013) 9(3):e1003233. doi: 10.1371/journal.ppat.1003233

\section{FUNDING}

This work was funded by NIH/NHLBI (R01 HL126076 and R01 HL132951) to JH, 5 K08 HL141446 to DG, and P01 HL132819 to JZ. It was also funded in part by NCI P30CA016359 and NIH/ NIDDK U54 DK106857 to Yale University.

\section{SUPPLEMENTARY MATERIAL}

The Supplementary Material for this article can be found online at: https://www.frontiersin.org/articles/10.3389/fimmu.2021.704072/ full\#supplementary-material

15. Merle NS, Noe R, Halbwachs-Mecarelli L, Fremeaux-Bacchi V, Roumenina LT. Complement System Part II: Role in Immunity. Front Immunol (2015) 6:257. doi: 10.3389/fimmu.2015.00257

16. Merle NS, Church SE, Fremeaux-Bacchi V, Roumenina LT. Complement System Part I - Molecular Mechanisms of Activation and Regulation. Front Immunol (2015) 6:262. doi: 10.3389/fimmu.2015.00262

17. Patel SR, Gibb DR, Girard-Pierce K, Zhou X, Rodrigues LC, Arthur CM, et al. Marginal Zone B Cells Induce Alloantibody Formation Following RBC Transfusion. Front Immunol (2018) 9:2516. doi: 10.3389/fimmu.2018.02516

18. Zerra PE PS, Jajosky RP, Arthur CM, McCoy JW, Allen JWL, Chonat S, et al. Marginal Zone B Cells Mediate a CD4 T Cell Dependent Extrafollicular Antibody Response Following Rbc Transfusion in Mice Blood. In press (2021). doi: 10.1182/blood.2020009376

19. Mener A, Patel SR, Arthur CM, Chonat S, Wieland A, Santhanakrishnan M, et al. Complement Serves as a Switch Between CD4+ T Cell-Independent and -Dependent RBC Antibody Responses. JCI Insight (2018) 3(22). doi: $10.1172 /$ jci.insight.121631

20. Das A, Heesters BA, Bialas A, O’Flynn J, Rifkin IR, Ochando J, et al. Follicular Dendritic Cell Activation by TLR Ligands Promotes Autoreactive B Cell Responses. Immunity (2017) 46(1):106-19. doi: 10.1016/j.immuni.2016.12.014

21. Stegmann TC, Veldhuisen B, Nagelkerke SQ, Winkelhorst D, Schonewille H, Verduin EP, et al. RhIg-Prophylaxis Is Not Influenced by FCGR2/3 Polymorphisms Involved in Red Blood Cell Clearance. Blood (2017) 129 (8):1045-8. doi: 10.1182/blood-2016-05-716365

22. Kumpel BM. Lessons Learnt From Many Years of Experience Using Anti-D in Humans for Prevention of RhD Immunization and Haemolytic Disease of the Fetus and Newborn. Clin Exp Immunol (2008) 154(1):1-5. doi: 10.1111/ j.1365-2249.2008.03735.x

23. Bowman JM, Pollock JM. Failures of Intravenous Rh Immune Globulin Prophylaxis: An Analysis of the Reasons for Such Failures. Transfus Med Rev (1987) 1(2):101-12. doi: 10.1016/s0887-7963(87)70010-8

24. Tampaki A, Gavriilaki E, Varelas C, Anagnostopoulos A, Vlachaki E. Complement in Sickle Cell Disease and Targeted Therapy: I Know One Thing, That I Know Nothing. Blood Rev (2021) 48:100805. doi: 10.1016/j.blre.2021.100805

25. Merle NS, Boudhabhay I, Leon J, Fremeaux-Bacchi V, Roumenina LT. Complement Activation During Intravascular Hemolysis: Implication for Sickle Cell Disease and Hemolytic Transfusion Reactions. Transfus Clin Biol (2019) 26(2):116-24. doi: 10.1016/j.tracli.2019.02.008

26. Floch A, Morel A, Zanchetta-Balint F, Cordonnier-Jourdin C, Allali S, Grall M, et al. Anti-C5 Antibody Treatment for Delayed Hemolytic Transfusion Reactions in Sickle Cell Disease. Haematologica (2020) 105(11):2694-7. doi: 10.3324/haematol.2020.253856

27. Calabro S, Gallman A, Gowthaman U, Liu D, Chen P, Liu J, et al. Bridging Channel Dendritic Cells Induce Immunity to Transfused Red Blood Cells. J Exp Med (2016) 213(6):887-96. doi: 10.1084/jem.20151720

28. Zhang L, Ding Z, Heyman B. IgG3-antigen Complexes are Deposited on Follicular Dendritic Cells in the Presence of C1q and C3. Sci Rep (2017) 7 (1):5400. doi: 10.1038/s41598-017-05704-3

29. Mener A, Patel SR, Arthur CM, Stowell SR. Antibody-Mediated Immunosuppression can Result From RBC Antigen Loss Independent of 
Fcgamma Receptors in Mice. Transfusion (2019) 59(1):371-84. doi: 10.1111/ trf. 14939

30. Yu H, Stowell SR, Bernardo L, Hendrickson JE, Zimring JC, Amash A, et al. Antibody-Mediated Immune Suppression of Erythrocyte Alloimmunization can Occur Independently From Red Cell Clearance or Epitope Masking in a Murine Model. J Immunol (2014) 193(6):2902-10. doi: 10.4049/jimmunol.1302287

31. Cruz-Leal Y, Marjoram D, Lazarus AH. Erythrocyte Saturation With IgG is Required for Inducing Antibody-Mediated Immune Suppression and Impacts Both Erythrocyte Clearance and Antigen-Modulation Mechanisms. J Immunol (2018) 200(4):1295-305. doi: 10.4049/jimmunol.1700874

32. Maier CL, Mener A, Patel SR, Jajosky RP, Bennett AL, Arthur CM, et al. AntibodyMediated Immune Suppression by Antigen Modulation is Antigen-Specific. Blood Adv (2018) 2(21):2986-3000. doi: 10.1182/bloodadvances.2018018408

33. Gruber DR, Richards AL, Howie HL, Hay AM, Lebedev JN, Wang X, et al. Passively Transferred IgG Enhances Humoral Immunity to a Red Blood Cell Alloantigen in Mice. Blood Adv (2020) 4(7):1526-37. doi: 10.1182/ bloodadvances.2019001299

34. Zwiers C, Koelewijn JM, Vermij L, van Sambeeck J, Oepkes D, de Haas M, et al. ABO Incompatibility and RhIG Immunoprophylaxis Protect Against Non-D Alloimmunization by Pregnancy. Transfusion (2018) 58(7):1611-7. doi: 10.1111/trf.14606

35. Cruz-Leal Y, Lazarus AH. Could Antigen Loss be a Potential Mechanism to Explain Antibody-Mediated Immune Suppression? Transfusion (2021) 61 (4):1004-6. doi: 10.1111/trf.16309
36. Tormey CA, Hendrickson JE. Transfusion-Related Red Blood Cell Alloantibodies: Induction and Consequences. Blood (2019) 133(17):182130. doi: 10.1182/blood-2018-08-833962

37. Kovács KG, Mácsik-Valent B, Matkó J, Bajtay Z, Erdei A. Revisiting the Coreceptor Function of Complement Receptor Type 2 (Cr2, CD21); Coengagement With the B-Cell Receptor Inhibits the Activation, Proliferation, and Antibody Production of Human B Cells. Front Immunol (2021) 12:620427. doi: 10.3389/fimmu.2021.620427

38. Zelek WM, Xie L, Morgan BP, Harris CL. Compendium of Current Complement Therapeutics. Mol Immunol (2019) 114:341-52. doi: 10.1016/ j.molimm.2019.07.030

Conflict of Interest: The authors declare that the research was conducted in the absence of any commercial or financial relationships that could be construed as a potential conflict of interest.

Copyright (๑) 2021 Escamilla-Rivera, Santhanakrishnan, Liu, Gibb, Forsmo, Foxman, Eisenbarth, Luckey, Zimring, Hudson, Stowell and Hendrickson. This is an openaccess article distributed under the terms of the Creative Commons Attribution License (CC BY). The use, distribution or reproduction in other forums is permitted, provided the original author(s) and the copyright owner(s) are credited and that the original publication in this journal is cited, in accordance with accepted academic practice. No use, distribution or reproduction is permitted which does not comply with these terms. 